\title{
Voriconazole in Treatment of Resistant Fungal Keratitis
}

\author{
Khalid Mehmood ${ }^{1}$, Sidrah Riaz ${ }^{2}$, Tariq Khan ${ }^{3}$, Mahfooz Hussain ${ }^{4}$, Sara Riaz ${ }^{5}$ \\ ${ }^{1}$ Avicenna Medical College, ${ }^{2-3}$ Akhtar Saeed Medical and Dental College, Lahore, ${ }^{4}$ Leading Reading Hospital, \\ Peshawar, ${ }^{5}$ Islam Medical College, Gujranwala
}

\begin{abstract}
Purpose: To study the effect of intrastromal Voriconazole for the treatment of resistant fungal keratitis in a tertiary care eye hospital in Lahore, Pakistan.
\end{abstract}

Study Design: Experimental interventional study.

Place and Duration of Study: Avicenna Medical College Hospital, Lahore, from July 2017 to July 2019.

Methods: Sixty four patients were selected. All patients with fugal keratitis were included. The patients with previous corneal scar, mature cataract, endophthalmitis, Panophthalmitis, scleral involvement, impending or frank corneal perforation and uncontrolled diabetic patients were excluded. Corneal scrapings of all patients were sent for $10 \% \mathrm{KOH}$ staining. All patients were given intrastromal Voriconazole at 3 to 4 sites in divided doses in one $\mathrm{ml}$ syringe with 27-guage needle. Injection was repeated on $4^{\text {th }}$ and $8^{\text {th }}$ day. It was combined by topical antifungal and antibiotic eye drops six hourly. Patients were followed at day two, five, nine, three weeks and at 3 months.

Results: There were 55 males and 9 females. Average size of ulcer was $6.4 \mathrm{~mm}$ ranging from $5.5 \mathrm{~mm} \pm 1.8 \mathrm{~mm}$. Fifty six (88\%) patients showed improvement while eight (12\%) patients ended up in melting of cornea which was managed with tectonic corneal graft. In three $(5 \%)$ patients penetrating Keratoplasty was done. Conjunctival congestion and ocular pain improved significantly one week after third dose but final visual acuity was not significantly improved due to scarring.

Conclusion: Intrastromal corneal voriconazole is an effective treatment for fungal keratitis in term of healing of the corneal ulcer, control of infection and prevention of corneal perforation and permanent blindness.

Key Words: Voriconazole, Fungal keratitis, Penetrating Keratoplasty.

How to Cite this Article: Mehmood K, Riaz S, Khan T, Hussain M, Riaz S. Variconazole in Treatment of Resistant Fungal Keratitis. Pak J Ophthalmol. 2020; 36 (4): 324-328.

Doi: https://doi.org/10.36351/pjo.v36i4.1002

\section{INTRODUCTION}

Fungal keratitis is challenging disease, which is difficult to diagnose and treat. Once colonized in the cornea, fungi have the propensity to penetrate deeper

Correspondence: Sidrah Riaz

Akhtar Saeed Medical and Dental College Lahore

Email:sidrah893@hotmail.com

Received: February 8, 2020

Accepted: July 20, 2020 corneal layers and if they get access to anterior chamber of eye then control of infection is extremely difficult. ${ }^{1}$ Cornea is an avascular structure and restricted defense mechanisms make it easy for fungi to colonize. These are usually found in soil, water and on plants. The important risk factors in developing fungal keratitis are trauma with vegetative material, contact lens wear and immunocompromised status. ${ }^{2,3,4,5}$ The prevalence of fungal keratitis is more in warm climate. Commonly implicated organisms in developing warm countries are Fusarium and Aspegillus. ${ }^{6,7}$ The rapid development of fungal 
keratitis leads to visual loss so early diagnosis is essential to prevent long term ocular complications. ${ }^{8}$ Poor outcome of fungal keratitis than bacterial keratitis is due to poor penetration and limited availability of antifungal drugs. ${ }^{9,10}$

Fungus was first documented in 1879 and accounts for 40 to $50 \%$ of all cases of keratits. ${ }^{11,12}$ There are some 70 different types of fungi but two are more relevant in ophthalmology which are yeast and filamentous fungi (septate and non-septate).

Fungal keratitis is very difficult to treat. Many a times, anti-fungal drugs given in the form of eye drops are not sufficient. The purpose of our study was to find out the effectiveness of intrastromal Voriconazole in the treatment of fungal keratitis.

\section{METHODS}

There were 64 patients included in the study. Study duration was from July 2017 to July 2019. All patients with clinical diagnosis of fugal keratitis were included either from outpatient department (OPD) or were referred from other medical centers. Patients'age, gender, size of ulcer and visual acuity were noted. The patients with previous corneal scar, mature cataract, endophthalmitis, Panophthalmitis, scleral involvement, impending or frank corneal perforation and uncontrolled diabetic patients were excluded. Clinical features of fungal keratitis included ocular pain, photophobia, decreased vision, satellite corneal lesions, corneal edema, haze and hypopyon. Corneal scrapingswere sent for $10 \% \mathrm{KOH}$ staining in all patients. Voriconazole is an antifungal drug available in $200 \mathrm{mg}$ vial, which needs dilution and once reconstituted it needs refrigeration. It can be used for 7 to 10 days after dilution. All patients were given intrastromal injection. The dose was $50 \mathrm{micro}-\mathrm{lit} / \mathrm{ml}$ at 3 to 4 sites, in divided doses, in clear cornea around the lesion. It produced hydration of corneal stroma around the lesion. One $\mathrm{ml}$ syringe with 27 guage needle was used for injection, with needle bevel down wards in corneal stroma. It was given at first, $4^{\text {th }}$ and $8^{\text {th }}$ day of presentation. Moderate to severe pain was common complaint by all patients which was treated by oral NSAIDS in all patients at the time of injection. Intrastromal injection was combined by topical antifungal, Voriconazole eye drops $1 \mathrm{mg} / \mathrm{ml}$ and antibiotic eye drops, Moxifloxacin $0.5 \%$ both QID for 4 weeks. Patients were followed-up on day two, five, nine, three weeks and 3 monthly post treatment. Visual acuity was noted at each visit. Patient was asked about pain and slit lamp examination was done to note size of lesion, resolution of hypopyon and epithelization of defect.

No systemic side effects of the drug were detected. No case of endophthalmitis or Panophthalmitis was observed. The patients were followed-up for 6 months.

\section{RESULTS}

Sixty four patients were selected, fifty five were males and nine were females. Mean age was $32 \pm 8$ years and age range was 18 to 60 years. Corneal scrapings were sent to lab for $10 \% \mathrm{KOH}$ staining. Only $37 \%$ showed positive staining and $63 \%$ were negative. Fifty six patients $(88 \%)$ responded well (47 males and 8

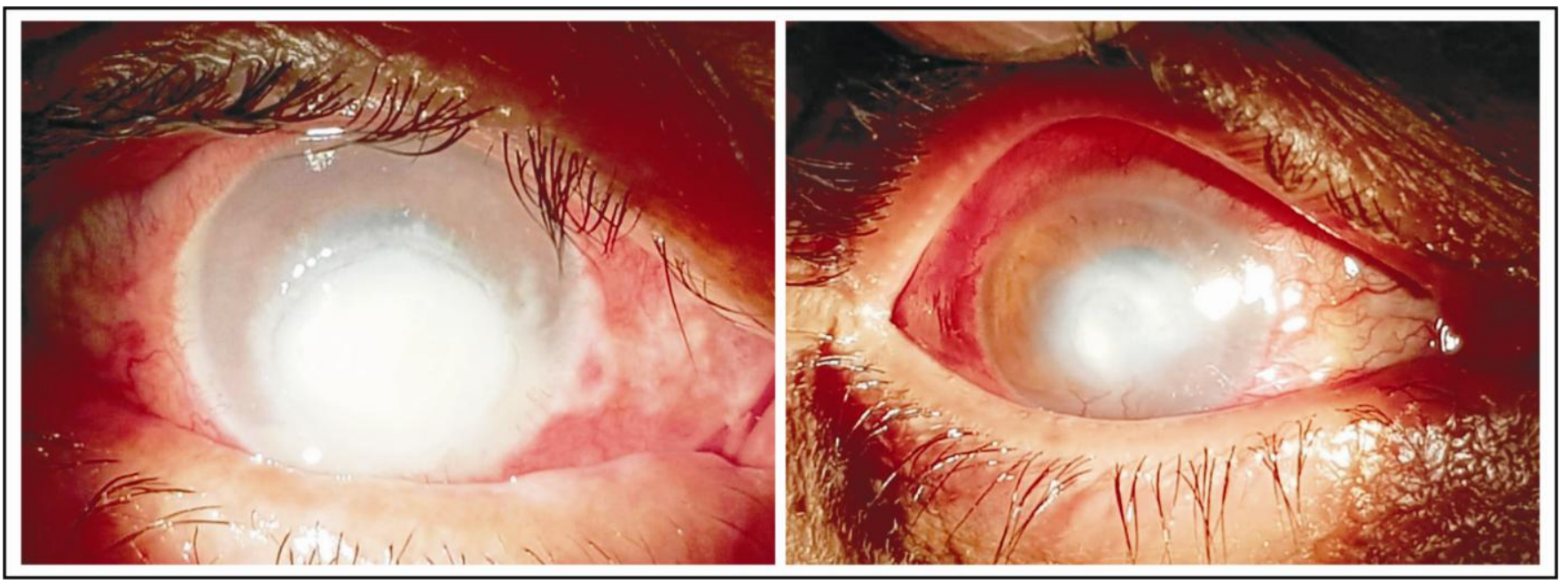

Fig. 1: Corneal Ulcer Before (Left) and After Treatment (Right). 


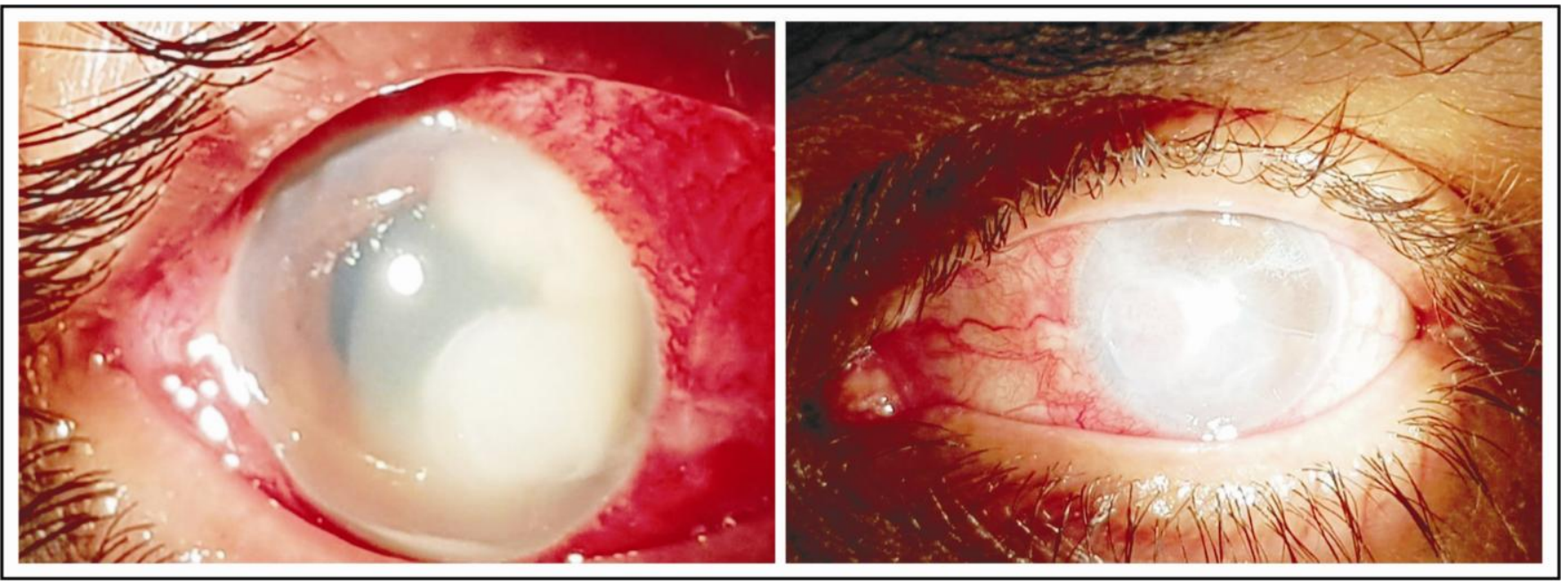

Fig. 2: Corneal Ulcer Before (Left) and After Treatment (Right).

females) to intrastromal Voriconazole, in terms of decrease in size of corneal infiltrates, improvement in ocular pain, resolution of corneal edema and healing of corneal ulcer with scarring of cornea. Eight (12\%) patients ( 7 males and 1 female) did not respond and ended up in melting of cornea which was managed with tectonic corneal graft. Out of these eight, only three patients required Penetrating Keratoplasty for visual restoration. Conjunctival congestion and ocular pain improved significantly one week after third dose of intrastromal Voriconazole but final visual acuity was not significantly improved due to scarring and most patients had counting finger vision after resolution of keratitis. No patient needed evisceration during 6 months follow-up period.

\section{DISCUSSION}

There are different anti-fungal drugs; which include, Polyenes, Imidazoles, Triazoles and Fluorinated Pyrimidines. ${ }^{13}$ Commonly used antifungal drugs are; Natamycin, Amphoteracin B, and Voriconazole. These drugs are used as topical and systemic therapy for treatment of fungal keratitis. We found intrastromal anti fungal drug delivery most effective as it achieved targeted drug delivery.

Higher incidence of fungal keratitis in male patients may be due to our social set up where males are more exposed to outdoors than women and it was comparable with other studies done by Al-Hatim et al and $\mathrm{CH}$ Cho et al. ${ }^{14,15}$

Ideally every corneal scraping should be sent for PCR and culture for diagnosis. PCR takes only two to three hours and culture takes up to 35 days. ${ }^{16} \mathrm{We}$ did not perform these tests in our study.

Natamycin 5\% eye drops belong to polyene group and it was the first approved antifungal agent. It inhibits transport of amino acids and glucose across fungal plasma membrane by binding with ergosterol leading to cell damage, but it is used only as a topical drug as negligible oral absorption makes it unfit for systemic drug. ${ }^{17}$ Voriconazole belongs to Triazole group, fungistatic and fungicidal, available for oral and parenteral use and metabolized in liver. It inhibits fungal cytochrome P-450 3A dependent enzymes and inhibits ergosterol synthesis, which is the principal sterol in cell wall of fungus and inhibits cell membrane synthesis. It is effective against Candida, Aspergillus, Fusarium, Scedosporium and Paecilomyces. It is effective in fungal keratitis resistant to Polyenes and first line Triazoles. It is also used as alternative to Amphoteracin B in fungal endophthalmitis.

Recent studies show that intrastromal use of voriconazole has produced better results in term of control of fungal infection and healing of ulcer. Ganapathy $\mathrm{K}$ showed that Intrastromal voriconazole helped to resolve the infection in $18(72 \%)$ patients and about $15 \%$ of these needed more than one injection. Smaller ulcers responded better to treatment. Fusarium species were responsible for six of the seven cases. ${ }^{18}$ According to Namrata Sharma, of 12 eyes, 10 eyes healed with scar formation, and the mean resolution time was $39.75 \pm 7.62$ days. Two corneas perforated and required therapeutic penetrating keratoplasty. ${ }^{19}$ Other studies also showed that Voriconazole has potential to achieve adequate drug 
concentration at the site of infection through a targeted drug delivery. ${ }^{20-22}$

Intrastromal Amphoteracin B is used in the same fashion as Voriconazole. Average healing time in our study was 21 days which was comparable with studies in other countries. A study in India by Kalaisselvi et al, showed mean resolution time of resistant fungal keratitis in 25 patients was 17 days. ${ }^{18}$ Another study by Sharma et al. showed healing time of $39 \pm 7$ days. ${ }^{19}$

In our study $5 \%$ patients showed no response to treatment and ended up as candidate of penetrating Keratoplasty. Literature shows that $30 \%$ patients with fungal keratitis develop corneal perforation or do not respond to topical antifungal therapy. ${ }^{23-25}$ Penetrating Keratoplasty is expensive treatment but if intraocular contents are not involved it can result in complete eradication of infection.

Limitation of our study are that we did not compare our results with any other antifungal drug, single center study and small sample size.

\section{CONCLUSION}

Our study showed that intrastromal Voriconazole is effective in fungal keratitis in term of good healing of corneal ulcer, control of infection, saving eye from corneal perforation, evisceration and loss of vision. No systemic side effects of drug were observed.

\section{Ethical Approval}

The study was approved by the Institutional review board/Ethical review board.

\section{Conflict of Interest}

Authors declared no conflict of interest.

\section{REFERENCES}

1. Gopinathan U, Sharma S, Garg P, Rao GN. Review of epidemiological features, microbiological diagnosis and treatment outcome of microbial keratitis: experience of over a decade. Ind J Ophthalmol. 2009; 57: 273-279.

2. Zapp D, Loos D, Feucht N, Khoramnia R, Tandogan T, Reznicek L, et al. Microbial keratitis-induced endophthalmitis: incidence, symptoms, therapy, visual prognosis and outcomes. BMC Ophthalmol. 2018; 18 (1): 112 .
3. Shimizu E, Yamaguchi T, Yagi-Yaguchi Y, Dogru M, Satake Y, Tsubota K, et al. Corneal Higher-Order Aberrations in Infectious Keratitis. Am. J. Ophthalmol. 2017; 175: 148-158.

4. Taneja MJ, Ashar N, Mathur A, Nalamada S, Grag P. Microbial keratitis following vegetative matter injury. Int Ophthalmol. 2013; 33 (2): 117-123.

5. Thomas PA, Kaliamurthy J. Mycotic keratitis: epidemiology, diagnosis and management. Clin Microbiol Infect. 2013; 19 (3): 210-220.

6. Foster CS. Fungal keratitis. Infect Dis Clin North Am. 1992; 6 (4): 851-857.

7. Wong TY, Ng TP, Fong KS, Tan DT. Risk factors and clinical outcomes between fungal and bacterial keratitis: a comparative study. CLAO J. 1997; 23: 275281.

8. Sharma S. Diagnosis of fungal keratitis: current options. Expert Opin Med Diagn. 2012; 6 (5): 449-455.

9. Florcruz NV, Evans JR. Medical Intervention for fungal keratitis. Cochrane Database Syst Rev. 2012; 2: 2015.

10. Lalitha P, Shapiro BL, Srinivasan M, Prajna NV, Acharya NR, Fothergill AW, et al. Antimicrobial susceptibility of Fusarium, Aspergillus, and other filamentous fungi isolated from keratitis. Archives of Ophthalmology, 2007; 125 (6): 789-793.

11. Tena D, Rodríguez N, Toribio L, GonzálezPraetorius A. Infectious Keratitis: Microbiological Review of 297 Cases. JPN. J. Infect. Dis. 2019; 72 (2): 121-123.

12. Mittal R, Ahooja H, Sapra N. Corneal Plaque formation after anti-acanthamoeba therapy in acanthamoeba keratitis. Indian J Ophthalmol. 2018; 66 (11): 1623-1624.

13. Bhartiya P, Daniell $\mathbf{M}$, Constantinou $\mathbf{M}$, Islam FMA, Taylor HR. Fungal keratitis in Melbourne. Clin Exp Ophthalmol. 2007; 35 (2): 124-130.

14. Al-Hatmi AMS, Castro MA, de Hoog GS, Badali H, Alvarado VF, Verweij PE, et al. Epidemiology of Aspergillus species causing keratitis in Mexico. Mycoses. 2019; 62 (2): 144-151.

15. Cho CH, Lee SB. Comparison of clinical characteristics and antibiotic susceptibility between Pseudomonas aeruginosa and $\mathrm{P}$. putida keratitis at a tertiary referral center: a retrospective study. BMC Ophthalmol. 2018; 18 (1): 204.

16. Ferrer C, Alio JL. Evaluation of molecular diagnosis in fungal keratitis Ten years of experience. Journal of ophthalmic inflammation and infection, 2011; 1: 15-22.

17. Farrell S, McElnea E, Moran S, Knowles S, Murphy CC. Fungal keratitis in the Republic of Ireland. Eye (Lond). 2017; 10: 1427-1434.

18. Kalaiselvi G, Narayana S, Krishnan T, Sengupta S. Intrastromal voriconazole for deep recalcitrant fungal keratitis: a case series. Br J Ophthalmol. 2015; 99 (2): 195-198. 
19. Sharma N, Agarwal $\mathbf{P}$, Sinha R, Titiyal JS, Velpandian T, Vajpayee RB. Evaluation of intrastromal voriconazole injection in recalcitrant deep fungal keratitis: case series. Br J Ophthalmol. 2011; 95 (12): 1735-1737.

20. Siatiri H, Daneshgar F, Siatiri N, Khodabande A. The effects of intrastromal voriconazole injection and topical voriconazole in the treatment of recalcitrant Fusarium keratitis. Cornea, 2011; 30 (8): 872-875.

21. Prakash G, Sharma N, Goel M, Titiyal JS, Vajpayee RB. Evaluation of intrastromal injection of voriconazole as a therapeutic adjunctive for the management of deep recalcitrant fungal keratitis. Am J Ophthalmol. 2008; 146 (1): 56-59.

22. Jones A, Muhtaseb M. Use of voriconazole in fungal keratitis. J Cataract Refract Surg. 2008; 34 (2): 183184. Doi: 10.1016/j.jcrs.2007.09.031.

23. Fontana L, Moramarco A, Mandarà E, Russello G, Iovieno A. Interface infectious keratitis after anterior and posterior lamellar keratoplasty. Clinical features and treatment strategies. A review. Br J Ophthalmol. 2019; 103 (3): 307-314.
24. McElnea E, Power B, Murphy C. Interface Fungal Keratitis after Descemet Stripping Automated Endothelial Keratoplasty: A Review of the Literature with a Focus on Outcomes. Cornea, 2018; 37 (9): $1204-$ 1211.

25. Xie L, Dong X, Shi W. Treatment of fungal keratitis by penetrating keratoplasty. Br J Ophthalmol. 2001; 85: 1070-1074.

\section{Authors' Designation and Contribution}

Khalid Mehmood; Professor: Concepts, Data Acquisition, Literature research, Manuscript preparation, Manuscript review.

Sidrah Riaz; Associate Professor: Design, Literature research, Statistical analysis, Manuscript preparation, Manuscript editing.

Tariq Khan; Professor: Literature research, Manuscript preparation, Manuscript review.

Mahfooz Hussain; Assistant Professor. Manuscript writing, Critical review.

Sara Riaz; Assistant Professor: Manuscript editing, Manuscript review. 